\title{
REFLEXIÓN SOBRE EL DISEÑO INTERIOR EN ESPACIOS EDUGATIVOS A PARTIR DE LAS INTELIGENGIAS MÚLTIPLES DE GARDNER
}

\section{REFLECTION ON INTERIOR DESIGN IN EDUCATIONAL SPACES BASED ON GARDNER'S MULTIPLE INTELLIGENGES}

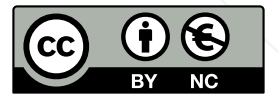

\author{
María Angélica Rosales Cisneros \\ Investigador independiente \\ Ecuador
}

Diseñadora de Interiores en la Universidad del Azuay, Ecuador (2020). La tesis de grado fue recomendada para la elaboración del presente artículo. Práctica profesional desde 2020 como diseñadora de interiores, en el ámbito comercial y educativo. A nivel académico ha recibido varias condecoraciones nacionales e internacionales, concursos internos de la Institución, ganadora del premio Manzaneidad 2020. Áreas de investigación: diseño educativo, relación diseño interior y arquitectura, metodologías de investigación.

mariangelica2597@gmail.com

orcid.org/0000-0003-3147-5837

\section{Manuela Cordero Salcedo Universidad del Azuay Ecuador}

Candidata a Doctora en Diseño por la Universidad de Palermo, Argentina, Diplomada en Antropología Social y Política por FLACSO Argentina, Magíster en Diseño por la Universidad del Azuay, Arquitecta por la Universidad de Cuenca. Docente e investigadora en la Universidad del Azuay, desde el año 2014. Entre las áreas de conocimiento en las que se ha involucrado están, la Gestión de la Cultura y el Patrimonio, la Antropología Cultural, estudios de la percepción relacionados a la movilidad en las ciudades.

manuelacordero@uazuay.edu.ec

orcid.org/0000-0001-5062-2447

Fecha de recepción: 28 de marzo, 2021. Aceptación: 20 de mayo, 2021. 


\section{Resumen}

El aprendizaje preescolar tiene como uno de sus objetivos facilitar el proceso de enseñanza, aplicando diversas metodologías en el aula de clase, con el propósito de reforzar el conocimiento y la inteligencia de los alumnos. Es así como busca fortalecer y fomentar el desarrollo inclusivo en niñas y niños para su óptimo desarrollo humano e intelectual en el espacio que acoge las actividades diarias de los establecimientos educativos, acompañando los objetivos de dichas instituciones. El presente artículo da cuenta de una reflexión respecto al diseño de espacios interiores para la educación primaria desde la mirada de las ocho Inteligencias Múltiples (IMG) del psicólogo y pedagogo Howard Gardner, este a su vez revisa y relaciona bibliografía sobre el trabajo de Gardner y propone alternativas para el diseño de espacios interiores que se correspondan con las IMG.

\section{Palabras clave}

Desarrollo infantil, educación inicial, espacios preescolares, Howard Gardner, IMG, inclusión.

\section{Abstract}

The objective of preschool learning is to facilitate the teaching process by applying various methodologies in the classroom to reinforce the knowledge and intelligence of the students. This is how preschool learning seeks to strengthen and promote inclusive development in girls and boys for their optimal human and intellectual development in the space that hosts students' daily activities, so that these institutions attain their objectives. This article gives a reflection of interior design spaces for primary education from Eight Multiple Intelligences perspective by the psychologist and pedagogue Howard Gardner (IMG); at the same time, it reviews and relates bibliography on Gardner's work and proposes alternatives for the design of interior spaces that correspond to the IMG.

\section{Keywords}

Child development, Early childhood education, Howard Gardner, Inclusion, IMG, Preschool spaces. 


\section{Introducción}

La teoría para educación inicial y sus herramientas están en constante revisión e innovación, ya que su propósito es mejorar el desarrollo de los y las estudiantes y su proceso de aprendizaje, favoreciendo la obtención de nuevos conocimientos en la etapa temprana de vida de los y las niñas. Las inteligencias múltiples de Gardner buscan fortalecer el desarrollo autodidacta por medio de la experimentación y el descubrimiento de las capacidades intelectuales y físicas de cada niño o niña. Es por esto que se lo relaciona directamente con características educativas lúdicas, inclusivas e interactivas. Este documento, que parte de investigación realizada en el trabajo de fin de carrera "Rediseño interior de espacios educativos a partir de las Inteligencias Múltiples de Gardner" (Rosales, Pintado y Cordero, 2020) propone criterios espaciales que se correspondan con los criterios pedagógicos propuestos por las IMG.

\section{Desarrollo}

La educación inicial se comprende como la atención o cuidado a niños y niñas desde el nacimiento hasta los seis años de edad, y su objetivo es fortalecer el desarrollo infantil a través de una atención integral que facilite el crecimiento y aprendizaje con aspectos físicos, cognitivos, socioemocionales, psicomotrices y del lenguaje. (Gil G y Sánchez G. 2004). Según el Ministerio de Educación del Ecuador (2019) los principios que se orientan a un desarrollo de educación inicial son: vinculación emocional y social, descubrimiento del medio natural y cultural, exploración del cuerpo y motricidad, manifestación del lenguaje verbal y no verbal, convivencia, identidad y autonomía, expresión artística, comprensión y expresión del lenguaje. La educación preescolar se la considera como el nivel más trascendental para el desarrollo en el proceso de aprendizaje de niñas y niños, por lo que es fundamental obtener habilidades que les permitan generar nuevos conocimientos. También se la considerada como el nivel más importante para el desarrollo en el proceso de aprendizaje de niñas y niños.
Se entiende, por otra parte, como espacio educativo al conjunto de aspectos que conforman un ambiente de aprendizaje y también donde se establece un encuentro educativo sistemático. Zabala (2020) propone que el aula de clase deberá estar organizada en distintas zonas de juego - trabajo, para fomentar el aprendizaje. Y a su vez, el aprendizaje autodidacta, es una característica de la educación inicial y se entiende como la manera en la que las personas forman su propio aprendizaje mediante la exploración y profundización de cualquier área del conocimiento. En este sentido, indican López, García, Chávez y Porras (2015) que un espacio educativo debe ser aprovechado para fomentar la autoformación de los alumnos. Un aprendizaje autodidacta aporta al desarrollo intelectual del niño, por lo que es importante estar atentos al comportamiento de los usuarios de un espacio educativo, para a partir de esta observación diseñar espacios que favorezcan el aprendizaje.

Autoras como Carla Ledesma (2012) en su artículo Uso y distribución de espacios escolares plantea cinco características a tomar en cuenta para crear un espacio educativo autodidacta (Ver Tabla 1). 
Tabla 1. Características de un espacio autodidacta propuestas por Ledesma (2012)

\begin{tabular}{|c|c|}
\hline & Características y criterios \\
\hline \multirow{5}{*}{$\begin{array}{l}\text { Características } \\
\text { de un espacio } \\
\text { educativo } \\
\text { autodidacta }\end{array}$} & Ampliable: Flexibilidad de ampliación \\
\hline & Convertible: Posibilidad de modificadores \\
\hline & $\begin{array}{l}\text { Polivalente: posibilidad de diferentes actividades en el } \\
\text { espacio }\end{array}$ \\
\hline & Variado: Garantía de un ambiente inclusivo e integral \\
\hline & Comunicable: intercambio de mobiliario \\
\hline
\end{tabular}

De Rosales, Pintado \& Cordero, (2020).

\section{Metodologías educativas en el aula de clase y las IMG}

Las metodologías educativas hacen y configuran el espacio educativo, indica Carmen Hernández (2018) que pueden clasificarse en tres tipos: expositiva, interactiva y de descubrimiento. Una metodología educativa tiene como propósito hacer que los estudiantes descubran un ambiente de trabajo dinámico y autodidacta. Howard Gardner ${ }^{1}$, señala que todos los seres humanos poseemos por lo menos ocho tipos de inteligencias potenciadas a través de la experimentación y la estimulación un el entorno de aprendizaje, de esta manera se puede fortalecer un ambiente educativo que desarrolle las habilidades en los estudiantes (Sartori, 2019).

Las inteligencias múltiples están directamente relacionadas con el mundo de la enseñanza y como lo señala Jáuregui Salas (2016) "Las inteligencias múltiples de Gardner son lenguajes que hablan todas las personas en parte por la cultura a la que cada una pertenece y constituyen herramientas que todos los seres humanos pueden utilizar para aprender a resolver problemas creando soluciones" (p. 12).
Sartori (2019) plantea que las inteligencias múltiples cambiarán según el proceso de crecimiento de cada persona, fortaleciendo la comprensión de una nueva inteligencia y ayudando a definir sus habilidades más afines. La inteligencia, como tal, se define como la facultad de la mente que está asociada con la capacidad de solucionar problemas, haciendo uso del razonamiento para adaptarse a diferentes ambientes y contextos (García, 2014).

Ximena Vélez Clavo (2005) considera a la inteligencia como el resultado de un proceso de un elemento dinámico o resultado de un pensamiento. Antiguamente se pensaba que la inteligencia del ser humano era de un solo tipo, clasificando a las personas como poco hábiles en ciertos temas o ciertos aprendizajes y muy hábiles en otros. Por esta razón, se realizaron varios estudios sobre diferentes consecuencias patológicas que son producto de lesiones del sistema nervioso y se concluyó, como lo señala H. Gardner, que el ser humano tiene ocho tipos de inteligencia (Vélez Clavo, 2005), que se muestran en la Tabla 2.

${ }^{1}$ Howard Gardner, psicólogo cognitivo, investigador y profesor de la universidad de Harvard, dedicó sus esfuerzos a investigar las capacidades cognitivas humanas para el avance de la calidad de la educación, basándose en el desarrollo y formación de las aptitudes de cada persona. En la década de los años 1980, publica The frames of mind: The Theory of Multiples Intelligences (1983), donde propone una metodología que servirá como aporte a la psicología y pedagogía (López, Dolabani, y Mosso, 2018). 
Tabla 2. Características de las Inteligencias múltiples de Gardner Interpretadas por Vázquez Seguí (2014)

\begin{tabular}{|c|c|}
\hline Inteligencia & Característica \\
\hline Lingüística & $\begin{array}{l}\text { Es la capacidad de usar las palabras de manera efectiva, } \\
\text { en forma oral o escrita. Incluye la habilidad en el uso de la } \\
\text { sintaxis, la fonética, la semántica y los usos pragmáticos } \\
\text { del lenguaje, así mismo consiste en usar el lenguaje para } \\
\text { expresar y entender significados complejos. }\end{array}$ \\
\hline Musical & $\begin{array}{l}\text { Es la capacidad de percibir, discriminar, transformar y } \\
\text { expresar las formas musicales. Además de incluir la } \\
\text { sensibilidad al ritmo, tono y timbre. }\end{array}$ \\
\hline $\begin{array}{l}\text { Lógica - } \\
\text { Matemática }\end{array}$ & $\begin{array}{l}\text { Esta inteligencia es un pensamiento lógico para entender } \\
\text { causa y efecto, conexión, relaciones entre acciones y } \\
\text { objetos e ideas. Así mismo tiene la habilidad para resolver } \\
\text { operaciones complejas, tanto lógicas como matemáticas. }\end{array}$ \\
\hline Espacial & $\begin{array}{l}\text { Está relacionada con la capacidad de pensar en tres } \\
\text { dimensiones, al percibir imágenes externas e internas, } \\
\text { recrearlas y transformarlas. Por otra parte, la persona que } \\
\text { desarrolle una memoria visual puede transformar temas } \\
\text { en imágenes, tal como se expresa en el arte gráfico. }\end{array}$ \\
\hline $\begin{array}{l}\text { Corporal - } \\
\text { kinestésica }\end{array}$ & $\begin{array}{l}\text { Es la capacidad de usar todo el cuerpo en la expresión de } \\
\text { ideas y sentimientos. Incluye habilidades de coordina- } \\
\text { ción, destreza, equilibrio, flexibilidad, fuerza y velocidad; } \\
\text { así también la capacidad kinestésica y la percepción de } \\
\text { medidas y volúmenes. }\end{array}$ \\
\hline Intrapersonal & $\begin{array}{l}\text { Se fundamenta en construir una percepción precisa } \\
\text { respecto de sí mismo y de organizar y dirigir su propia } \\
\text { vida. Ayuda a reflexionar y controlar los pensamientos y } \\
\text { sentimientos efectivamente }\end{array}$ \\
\hline Interpersonal & $\begin{array}{l}\text { Tiene la capacidad de entender a los demás e interactuar } \\
\text { eficazmente con ellos. Incorpora sensibilidad a las } \\
\text { expresiones faciales, la voz, los gestos y posturas. }\end{array}$ \\
\hline $\begin{array}{l}\text { Naturalística - } \\
\text { Pictórica }\end{array}$ & $\begin{array}{l}\text { Consiste en distinguir, clasificar y utilizar elementos del } \\
\text { medio ambiente, objetos, animales o plantas, igualmente } \\
\text { consiste en las habilidades de observación, experimenta- } \\
\text { ción, reflexión y cuestionamiento del entorno. }\end{array}$ \\
\hline
\end{tabular}

De Rosales, Pintado \& Cordero, (2020).

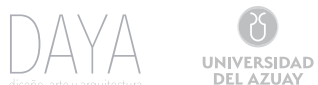


Como lo señala Sartori (2019), la educación juega un papel fundamental en la formación y desarrollo de las inteligencias de las personas, por lo que es necesario contar con ambientes que las favorezcan, es por esto que propone actividades que ayuden al desarrollo de cada una de las inteligencias propuestas por Gardner (Ver Tabla 3).

Tabla 3. Actividades sugeridas para fomentar un tipo de inteligencia según Sartori (2019)

\begin{tabular}{|l|l|}
\hline Inteligencia & Actividad sugerida \\
\hline Lingüística & $\begin{array}{l}\text { Fomentar la lectura, escritura y narración de historias, } \\
\text { cuentos, poemas, discursos, cartas, entre otros. }\end{array}$ \\
\hline Musical & $\begin{array}{l}\text { Usar música a medida que se estudia o se realiza la } \\
\text { actividad, relajarse previo a comenzar con el estudio. }\end{array}$ \\
\hline Lógico - & $\begin{array}{l}\text { Impulsar el uso de rompecabezas, juegos lógicos y crear } \\
\text { visitas a museos, exhibiciones, ferias científicas. }\end{array}$ \\
\hline Espacial & $\begin{array}{l}\text { Jugar con laberintos, rompecabezas, ajedrez y modelos } \\
\text { 3D con juegos de armar y desarmar. }\end{array}$ \\
\hline $\begin{array}{l}\text { Corporal - } \\
\text { kinestésico }\end{array}$ & $\begin{array}{l}\text { Establecer técnicas de movimientos como: danza, depor- } \\
\text { tes y movimientos creativos. }\end{array}$ \\
\hline Intrapersonal & $\begin{array}{l}\text { Respetar la rapidez de aprendizaje de cada estudiante. } \\
\text { permitiéndole superar sus propios tiempos y desempe- } \\
\text { ños anteriores. }\end{array}$ \\
\hline Naturalística & $\begin{array}{l}\text { Crear actividades de cooperación, de solución de dificulta- } \\
\text { des y juegos en parejas o en grupos, así sea de mesa 0 } \\
\text { competencias al aire libre. }\end{array}$ \\
\hline Interpersonal & $\begin{array}{l}\text { Hacer prácticas en las cuales se posibilite explorar los } \\
\text { sores vivientes, entendiendo, discriminando e identifican- }\end{array}$ \\
\hline
\end{tabular}

De Rosales, Pintado \& Cordero, (2020). 
Las actividades planteadas por Sartori (2019) permiten repensar las necesidades en las aulas educativas a fin de generar espacios que potencien el desarrollo de las IMG.

El diseño interior y su relación con el espacio educativo inicial, una revisión a los conceptos de espacios lúdicos y autodidactas

El diseño interior es una disciplina proyectual que trabaja sobre la generación de experiencias en el espacio interior, a través de la compresión del volumen espacial, y el tratamiento de sus superficies (Esdima, 2010). También se entiende como la actividad que busca generar sensaciones y percepciones de un área intervenida, conformando espacios que conjugan realidades psicológicas, económicas, ambientales, estéticas, funcionales y psicosociales, etc. En el caso de los espacios educativos, como señala Zabala (2020) es importante considerar que, tanto el mobiliario como los espacios deben resultar accesibles para los niños y niñas, para que puedan usarlos sin intermediarios y se favorezcan un ambiente lúdico y autodidacta. Además, fortalecer su desarrollo integral, permitirá a la o el profesor mayor libertad de acción para trabajar.

De igual manera, la sensibilidad estética de un aula es fundamental ya que un ambiente organizado y agradable ayuda a la estancia de los y las estudiantes. Asimismo, los colores llamativos y atractivos para los y las niñas llamarán su atención relacionándolos a determinados temas o contenidos, además también está demostrado que ciertos colores pueden estimular nuestra mente.

Los espacios de centros educativos preescolares deben ser esencialmente polivalentes ${ }^{2}$, y flexibles en su uso (Zabala, 2020), ya que deberán cumplir unos requisitos mínimos planteados por los organismos que regentan en el aula de clase.
Jaramillo (2013) expresa que, El espacio interior escolar debe estar diseñado para rendir al máximo el potencial académico, ya que los niños a su temprana edad despiertan el sentido exploratorio, es por esto que debe estar dispuesto de una forma donde los estudiantes puedan explorar por sí solos, usando juguetes y materiales autodidactas (p. 15).

En este sentido, todos los elementos que conforman el espacio escolar deben estar pensados para generar ambientes de inclusión. Por otra parte, como lo señala Brougere (2013) los espacios educativos al igual que el ambiente lúdico, deben estar basados en criterios que permitan a niños y niñas ser creativos e interactivos con su propio aprendizaje, deben siempre ir cambiando en función de fortalecer las metodologías de aprendizaje. Es indispensable incorporar en los espacios de aprendizaje actividades lúdicas que orienten y ayuden a lograr una inclusión pedagógica fomentando la interacción entre los miembros del aula para formar una relación empática entre el docente y el alumno mediante su diseño interior. Como señala Vivar (2011) la creación de un espacio interactivo requiere de la interrelación entre estímulos: movimiento, sonido, gestos o palabras, de esta forma se trata de generar un equilibrio entre las acciones y respuestas del cerebro al realizar cualquier actividad en el espacio.

Relación de las Inteligencias múltiples de Gardner como metodología y el diseño interior en un aula educativa inicial

A partir de la revisión del libro Lectura temprana y desarrollo del potencial intelectual del niño preescolar (2005) y una entrevista con su autora Ximena Vélez Calvo, la investigación de Rosales, Pintado \& Cordero (2020) realizó un cuadro de sugerencias y actividades para un aula educativa inicial en relación a las IMG (Ver Tabla 4).

${ }^{2}$ Polivalente: Hace referencia a las posibilidades de utilización que ofrecen las distintas zonas del aula (Zabala, 2020).

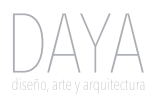


Las ocho inteligencias se encuentran presentes en variadas proporciones en todas las personas. Por ejemplo, "un niño puede tener una inteligencia lingüística y musical elevada, una inteligencia lógico matemática y naturalista intermedia, ser muy hábil interpersonalmente, pero muy limitado intrapersonalmente. Esta teoría evidencia las tendencias de aprendizaje y desempeño que tienen los seres humanos y cómo podemos descubrirlas y potenciarlas" (Vélez, 2005, p.17).

En este sentido, las inteligencias múltiples dominantes tienen una mayor fortaleza y se pueden determinar en los estilos de comportamiento y las preferencias de aprendizaje de niños y niñas.

Tabla 4. Sugerencias y actividades para un aula educativa inicial en relación a las IMG construida a partir de la revisión de Vélez (2005) y Sartori (2019)

\begin{tabular}{|c|c|c|}
\hline Inteligencia & $\begin{array}{l}\text { Sugerencias para la aplicación } \\
\text { de las Inteligencias múltiples } \\
\text { en el aula educativa inicial }\end{array}$ & $\begin{array}{l}\text { Actividades sugeridas para fomentar } \\
\text { las inteligencias múltiples dentro del } \\
\text { aula de clase }\end{array}$ \\
\hline Musical & $\begin{array}{l}\text { Música para ambientar } \\
\text { espacios y relajarse. }\end{array}$ & $\begin{array}{l}\text { Actividades con música o entonación, } \\
\text { usando sonidos, cintas o artefactos } \\
\text { musicales. }\end{array}$ \\
\hline $\begin{array}{l}\text { Lógico - } \\
\text { Matemático }\end{array}$ & $\begin{array}{l}\text { Repetición de series y } \\
\text { clasificación de tamaño, forma, } \\
\text { color, raza, texturas, etc. de objetos. }\end{array}$ & $\begin{array}{l}\text { Actividades de investigación, estudio } \\
\text { estadístico, juegos lógicos. }\end{array}$ \\
\hline Espacial & $\begin{array}{l}\text { Ilustrar, dibujar, pintar, } \\
\text { esculpir, construir. Usar color, forma } \\
\text { o imágenes en sus trabajos. }\end{array}$ & $\begin{array}{l}\text { Actividades con modelos gráficos, mapas o } \\
\text { juegos creativos, utilizando varios colores y } \\
\text { maneras para ordenar figuras. }\end{array}$ \\
\hline $\begin{array}{l}\text { Corporal - } \\
\text { kinestésico }\end{array}$ & $\begin{array}{l}\text { Crear y reforzar con } \\
\text { material manipulable. }\end{array}$ & $\begin{array}{l}\text { Juegos de papeles y drama, usando } \\
\text { herramientas y materiales manipulables, y } \\
\text { ocupar técnicas de movimientos creativos. }\end{array}$ \\
\hline Intrapersonal & Trabajos en grupo. & $\begin{array}{l}\text { Actividades de meditación e idealización } \\
\text { de proyectos o creaciones. } \\
\text { Respetar la rapidez del aprendizaje del } \\
\text { estudiante. }\end{array}$ \\
\hline Interpersonal & $\begin{array}{l}\text { Descripción de cualidades } \\
\text { y sentimientos a través del dibujo. }\end{array}$ & $\begin{array}{l}\text { Actividades de cooperación y solución de } \\
\text { dificultades. } \\
\text { Proponer actividades sociales y académicas. }\end{array}$ \\
\hline Naturalística & Exploraciones y excursiones. & $\begin{array}{l}\text { Impulsar la exploración, actividades al aire } \\
\text { libre y el respeto a la naturaleza. }\end{array}$ \\
\hline
\end{tabular}

De Rosales, Pintado \& Cordero, (2020).

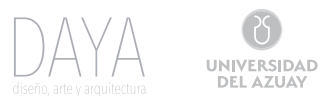


Factores conceptuales espaciales relacionados con las Inteligencias Múltiples de Gardner

Como estrategia para el diseño interior de espacios educativos, se relaciona las características de los espacios educativos planteadas por Ledesma (2012) y las Ocho IMG de H. Garder (Ver Figura 1).

Figura 1. Estrategias de diseño

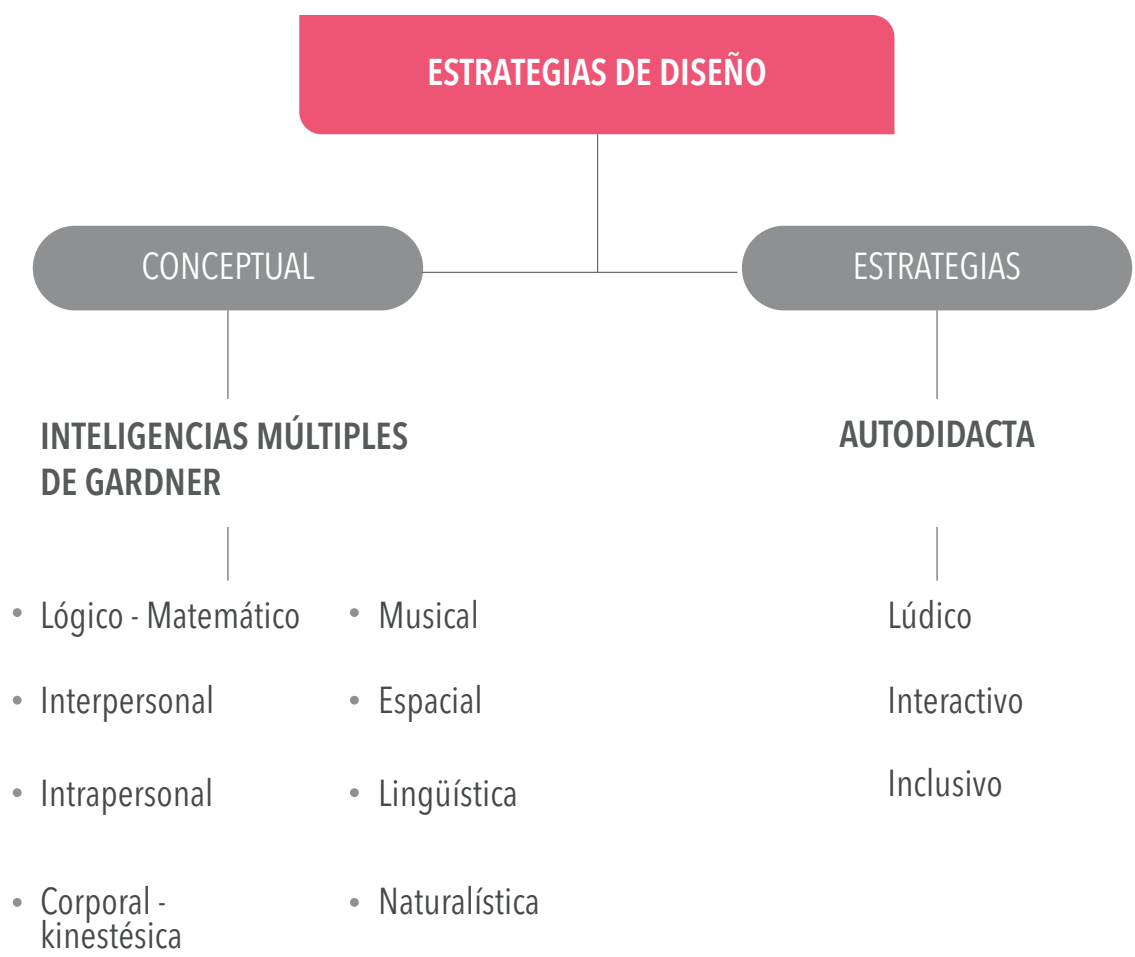

De Rosales, Pintado \& Cordero, (2020).

En base a las actividades propuestas por Sartori (2019) para potenciar las IMG y las sugerencias realizadas por Vélez (2005) para la aplicación de las IMG, se realizó un análisis de espacios para la educación inicial y se configuró un cuadro de Criterios conceptuales espaciales, que relaciona cada una de las inteligencias planteadas por Gardner (1983) con un espacio educativo (Ver Tabla 5). 
Tabla 5. Relación de espacios con las Inteligencias Múltiples de Gardner

RELACIÓN DE ESPACIOS CON LAS INTELIGENCIAS MÚLTIPLES DE GARDNER

\begin{tabular}{|c|c|c|c|}
\hline $\begin{array}{l}\text { Lógico - } \\
\text { Matemático }\end{array}$ & Interpersonal & Intrapersonal & Corporal - Kinestésico \\
\hline $\begin{array}{l}\text { Uso del espacio } \\
\text { para la exploración, } \\
\text { clasificación, } \\
\text { análisis y repetición } \\
\text { de habilidades } \\
\text { lógico cognitivas }\end{array}$ & $\begin{array}{l}\text { Área social inclusiva } \\
\text { para impulsar la } \\
\text { reflexión o medita- } \\
\text { ción y cooperación } \\
\text { en la solución de } \\
\text { problemas }\end{array}$ & $\begin{array}{l}\text { Área de concentra- } \\
\text { ción, autoevaluación } \\
\text { y estudio individual } \\
\text { del aprendizaje }\end{array}$ & $\begin{array}{l}\text { Elementos de } \\
\text { descubrimiento y } \\
\text { refuerzo del } \\
\text { lenguaje corporal } \\
\text { de tipo lúdico }\end{array}$ \\
\hline Musical & Espacial & Lingüística & Naturalística \\
\hline $\begin{array}{l}\text { Componentes e } \\
\text { instrumentos } \\
\text { musicales para la } \\
\text { ambientación, } \\
\text { memorización y } \\
\text { composición de } \\
\text { sonidos }\end{array}$ & $\begin{array}{l}\text { Zona de representa- } \\
\text { ción creativa de la } \\
\text { realidad a través de } \\
\text { la percepción visual } \\
\text { y espacial }\end{array}$ & $\begin{array}{l}\text { Espacio formal para } \\
\text { el manejo descriptivo } \\
\text { e informativo de la } \\
\text { expresión escrita y } \\
\text { oral }\end{array}$ & $\begin{array}{l}\text { Área verde interacti- } \\
\text { va para la explora- } \\
\text { ción del entorno con } \\
\text { fines recreativos, } \\
\text { sensitivos y } \\
\text { medioambientales }\end{array}$ \\
\hline
\end{tabular}

De Rosales, Pintado \& Cordero, (2020).

Una vez relacionadas las IMG con los espacios educativos, el trabajo de Pintado, et. al., (2020), propone una matriz en la que se establecen elementos y materiales que pueden configurar cada uno de los espacios educativos según las IMG con las que estos se relacionen sabiendo que estos espacios deberán también corresponder al desarrollo autodidacta, lúdico, inclusivo e interactivo de las y los niños que acoge (Ver Tabla 6). 
Tabla 6. Análisis de estrategias de diseño

ANÁLISIS ESTRATEGIAS DE DISEÑO

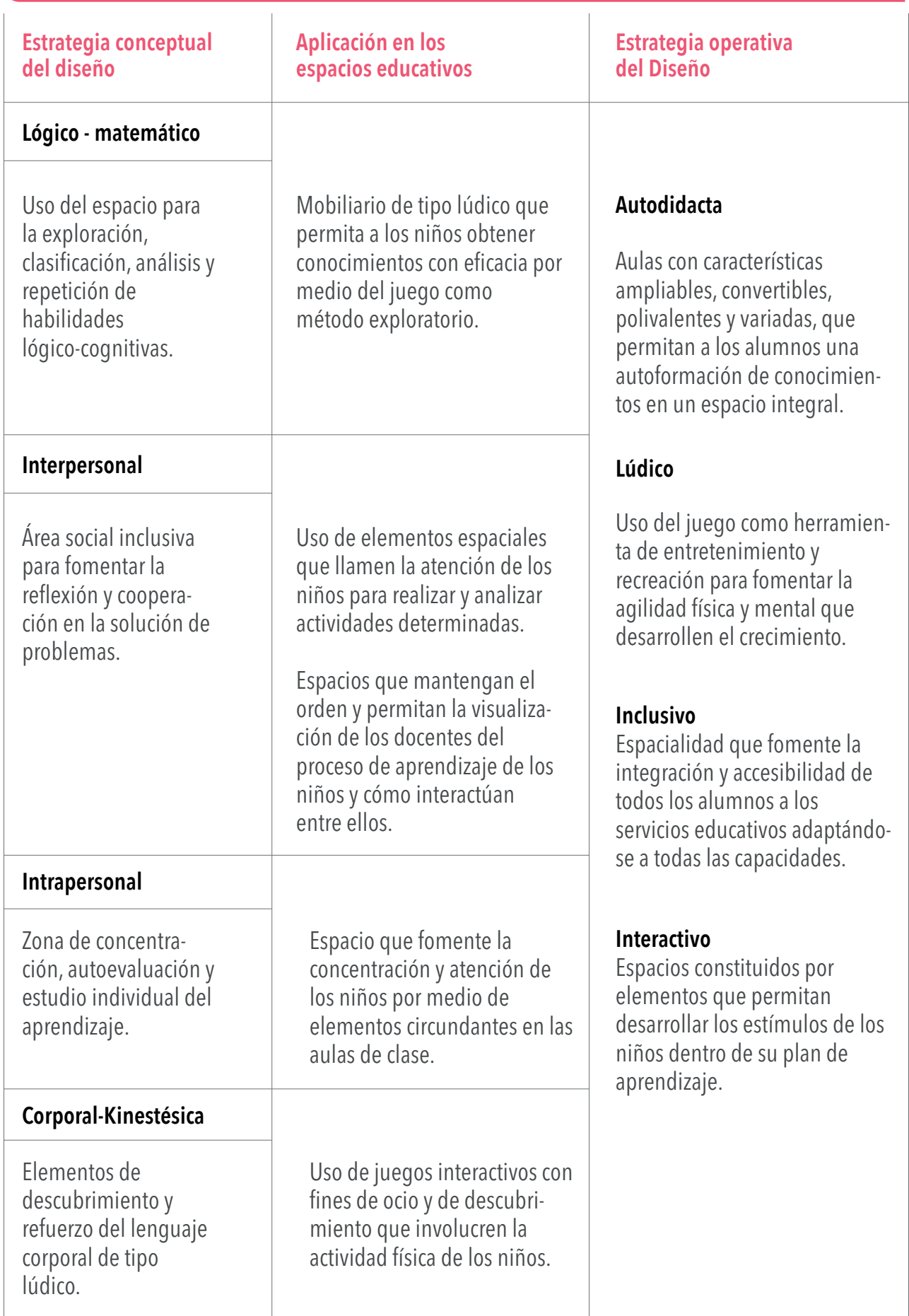




\begin{tabular}{|c|c|}
\hline Musical & \\
\hline $\begin{array}{l}\text { Componentes e } \\
\text { instrumentos musicales } \\
\text { para la ambientación, } \\
\text { memorización y } \\
\text { composición de sonidos. }\end{array}$ & $\begin{array}{l}\text { Uso de la música para } \\
\text { ambientar el entorno } \\
\text { educativo, y fomentar el } \\
\text { aprendizaje creativo y la } \\
\text { memoria de los niños. }\end{array}$ \\
\hline Espacial & \\
\hline $\begin{array}{l}\text { Zona de representa- } \\
\text { ción creativa de la } \\
\text { realidad a través de la } \\
\text { percepción visual y } \\
\text { espacial. }\end{array}$ & $\begin{array}{l}\text { Elementos espaciales lúdicos } \\
\text { que permitan a los niños } \\
\text { desarrollar un aprendizaje } \\
\text { autodidacta por medio de su } \\
\text { expresión creativa. }\end{array}$ \\
\hline Lingüístico & \\
\hline $\begin{array}{l}\text { Espacio formal para el } \\
\text { manejo descriptivo e } \\
\text { informativo de la } \\
\text { expresión escrita y } \\
\text { oral. }\end{array}$ & $\begin{array}{l}\text { Espacios conceptualmente } \\
\text { funcionales para el manejo de } \\
\text { la expresión oral (oratoria) y } \\
\text { mobiliario ergonómico para } \\
\text { facilitar la motricidad de los } \\
\text { niños. }\end{array}$ \\
\hline Naturalístico & $\begin{array}{l}\text { Espacios al aire libre que } \\
\text { conecten a los niños con la }\end{array}$ \\
\hline $\begin{array}{l}\text { Área verde interactiva } \\
\text { para la exploración del } \\
\text { espacio con fines } \\
\text { recreativos, sensitivos } \\
\text { y medioambientales. }\end{array}$ & $\begin{array}{l}\text { naturaleza como un entorno } \\
\text { que comprende el descubri- } \\
\text { miento de sensaciones y } \\
\text { sentidos. } \\
\text { Uso de vegetación dentro del } \\
\text { espacio para conectarlo con la } \\
\text { naturaleza. }\end{array}$ \\
\hline
\end{tabular}

De Rosales, Pintado \& Cordero, (2020). 


\section{Conclusiones}

El entorno educativo inicial está en constante cambio y actualización, y tal como se ha revisado en este documento, el entorno de aprendizaje forma parte fundamental del proceso de aprendizaje de niños y niñas. En este sentido un adecuado diseño de los espacios educativos requiere una cuidadosa investigación también sobre las metodologías de educación a fin de que los espacios se correspondan con sus objetivos. La relación entre la educación inicial y el diseño del espacio educativo, debe conseguir configurar no solo espacios contenedores, sino espacios que a través de sus elementos y la relación de sus usuarios con estos potencien la adquisición de nuevos conocimientos.

Las inteligencias múltiples se basan en experimentación de estímulos a través del descubrimiento de diferentes habilidades de pensamiento y capacidades del cerebro, su investigación ayuda a formular estrategias que contribuyen al entorno preescolar, que tiene como objetivo promover el autoaprendizaje temprano de niños y niñas. La revisión de las ocho inteligencias múltiples de Gardner, a la luz de los problemas que plantea el diseño interior de espacios educativos, permite repensar el papel que el espacio juega como articulador del aprendizaje, para la construcción de entornos educativos lúdicos e interactivos que impulsen un aprendizaje autodidacta. Este trabajo aporta a la reflexión sobre la importancia del diseño interior del espacio educativo y el amplio camino que abre hacia la exploración de las estrategias pedagógicas como punto de partida desde el cual se puedan plantear más y mejores espacios para el aprendizaje.

\section{Referencias}

Brougere, C. (2013). El Niño En La Cultura Lúdica. (pp:1-11)

Esdima. (2010). ¿Qué es el Diseño de Interiores? (pp: 94, 1-16). https://eurlex.europa.eu/LexUriServ/LexUriServ.do?uri=COM:2001:0179:FIN:ES:PDF

Gil, G. \& Sánchez, O. (2004). Educación Inicial o Preescolar: El Niño y La Niña Menores de Tres Años. Algunas Orientaciones a Los Docentes. Revista Venezolana de Educación. (pp: 27,535-543).

Hernández, C. (2018). Metodologías de enseñanza y aprendizaje en altas capacidades. (pp:13-20) Jaramillo, M. (2013). Diseño de Un Ambiente Preescolar Óptimo. (pp.21).

Jáuregui, S. (2016). La teoría de las inteligencias múltiples ¿práctica docente en la educación del Perú? Educación, (pp. 22, 87-91). https://doi.org/10.33539/educacion.2016.n22.1154

Ledesma, C. (2012). Uso y Distribución de Espacios Escolares. (pp.49).

López, J., García, S., Chávez, 0. \& Porras, S. (2015). Autodidactismo: ¿Una alternativa para una educación de calidad? (pp.41).

Ministerio de Educación del Ecuador. (2019). Currículo de Educación Inicial - Ministerio de Educación. Obtenido de: (https://educacion.gob.ec/educacion-inicial/).

Rosales, M., Pintado, M., \& Cordero, M. (2020). Rediseño Interior de Espacios Educativos a Partir de Las Inteligencias Múltiples de Gardner(IMG).http://dspace.uazuay.edu.ec/handle/datos/9923

Sartori, 0. (2019). Las Inteligencias Múltiples En El Aula de Clase. Obtenido de: Revista Internacional de Humanidades. (pp:63-77). https://doi.org/10.18848/2474- 5022/cgp/v06i01/63-77

Vázquez Seguí, N. (2014). La Teoría de Las Inteligencias Múltiples de Howard Gardner. (pp: 56-62)

Vélez, X. (2005). Lectura temprana y desarrollo del potencial del niño preescolar. (pp.183)

Vivar, R. (2011). Análisis y Sensibilización Del Diseño Con El Uso de Formas. (pp:41-42,50)

Zabala, M. (2020). Calidad en la educación infantil. (pp:45- 63) 